\title{
LA NORMA Y LA PRODUCCIÓN DEL LENGUAJE
}

\section{INTRODUCCIÓN}

Desde el punto de vista lingüístico, no existen parámetros absolutos de corrección dentro de una lengua. Cuando se afirma que tal o cual enunciado es agramatical o inapropiado, se conduye que quien lo produjo no posee el conocimiento lingüís tico de un hablante nativo, o que se están manejando reglas inmanentes en una variedad de la lengua que no corresponde a las reglas de la variedad que se pretende utilizar. De este modo, lo que es gramatical en una lengua puede no serlo en otra, y lo que es apropiado para una comunidad lingü ística, donde se usa un determinado dialecto regional o social, puede no serlo para otra.

En esta medida, las descripciones lingüísticas tienen como tarea descubrir y registrar, de la manera más objetiva posible, las reglas que maneja una comunidad de hablantes en el uso cotidiano de su lengua, y no busca imponer en ellos reglas de corrección para su comportamiento lingüístico.

Frente a la altemativa: descripción o prescripción, resulta evidente que la lingüística ha elegido la primera. Sin embargo, al preferir trabajar con las reglas inmanentes al funcionamiento de la lengua en una comunidad, esta ciencia no está sugiriendo que no haya lugar para la determinación de fómulas de corrección en su uso. De hecho, la teoría lingüística ha creado y desarrollado un concepto que corresponde, a grandes rasgos, a un parámetro que permite deteminar niveles de corrección en el uso de la lengua; el concepto de norma.

En su presentación de la noción en cuestión, Eugenio Coseriu [1982-94] nos dice:

....el individuo crea su expresión en una lengua, habla una lengua, realiza concretamente en su hablar moldes, estructuras de la lengua de su comunidad.

En un primer grado de formalización, esas estructuras son simplemente normales y tradicionales en la œmunidad, œnstituyendo lo que llamamos norma; pero en un plano de abstracción más al to, se desprende de ellas mismas una serie de elementos esenciales e indispensables de oposiciones funcionales; lo que llamamos sistema. (El subrayado es nuestro).

Existe pues, una diferencia clara entre la lengua como sistema de signos y la lengua como conjunto de estructuras nomales para una determinada comunidad. Es dentro de esta última perspectiva que podemos hablar de corrección o incorrección; un enunciado cualquiera será incorrecto si posee una forma "anormal" para las circunstancias en que se produce.

Dado que para hablar de norma necesariamente debe hablarse de comunidad lingüís tica y de contextos de uso de una lengua, ha sido dentro de la sociolingüística que tal concepto se ha analizado en mayor profundidad.

\footnotetext{
*Profesora de Lingüística de la Universidad Pedagógica Nacional. 
Se dice, por ejemplo, que dentro de una comunidad de hablantes puede existir una variedad de normas (familiar, literaria, culta, vulgar, etc.), en la medida en que existen también diferentes contextos de utilización de la lengua. Muchos sociolingüistas han trabajado dentro de esta área, describiendo la variación lingüística como un problema de lo que es nomal en unos casos y anormal en otros.

Además, se han adelantado investigaciones que estudian el problema de las actitudes de los hablantes frente a las nom as. Al respecto, Rubén Arboleda [1986 :67] explica:

La sociedad ha adquirido creencias y actitudes frente a las distintas normas, que se traducen en la valoración de éstas a la luz de parámetros como culta-inculta, correcta-incorrecta y en la prescripción de unas y la acción de privilegiar otras.

De esta manera la sociolingüística ha introducido las nociones de "variedad estándar" y "variedad vernácula". Mientras que a la primera se le asignan funciones integradoras, a la segunda se le atribuyen funciones diferenciadoras que resaltan las particularidades de la comunidad que la habla. Mientras que sobre la primera recaen con frecuencia juicios de valor positivos, la segunda, al tener una libertad contextual mucho menor, tiende a ser estigmatizada por los hablantes.

La sociolingüística se ha aproximado también al concepto de noma, a partir del estudio de la competencia comunicativa del hablante. Este, además de poseer un conocimiento del sistema - competencia lingüís tica - , posee un conocimiento de aquello que es apropiado en una deteminada situación de habla. forma:

Norbert Dittmar [1976:163] define competencia comunicativa de la siguiente

... la habilidad de los individuos para cornunicarse entre sí bajo condiciones (de naturaleza lingüística, psicológica, social y pragmática) definida situacional y normativamente.

As í, hablar una lengua implica, entre otras cosas saber utilizar las estructuras y el vocabulario adecuados en el momento adecuado. Lucía Tobón de Castro [1990 : 47] explica la idea de la siguiente manera:

En los procesos de producción e interpretación de unidades discursivas el hablante aplica no sólo la noma lingüística, como lo propone Coseriu, sino también una noma psico-sociopragmática, puesto que la lengua, como todo sistema semiológiø, cumple una función que permite a un individuo desarrollarse como persona por tanto sabe cómo actuar en una determinada situación y qué formas lingüísticas tiene que utilizar en el entorno en que se encuentra.

No queda duda, pues, de la existencia de las nomas y su operatividad en el plano social, reflejada en las actitudes lingüísticas y en el plano psicológico, reflejada en el comportamiento lingüístico del hablante. 
Sin embargo, queda todavía por examinar un enfoque de anális is que has ta el momento ha sido poco explorado: el de la noma como entidad mental que opera en los procesos psicolingüís ticos de producción y recepción del lenguaje. Desde este punto (le vis ta, la pregunta o el problema que debe resolverse, no es tanto si existe o no una noma, sino qué proceso mental cumple la función nomativa presente en todo comportamiento lingüís tico.

Interesadas en el tema, especialmente en la pregunta en cuestión, realizamos, en compañía de la profesora Amparo Leyva Z., un trabajo de búsqueda bibliográfica y de campo que nos permitiera llegar a infomaciones válidas para responder el interrogante planteado.

El trabajo se dividió en dos partes: en primer lugar, realizamos un estudio de la literatura psicolingüística, particulamente en el área de la producción del lenguaje ${ }^{1}$, que nos condujo a la fomulación de la hipótesis, y en segundo lugar llevarnos a cabo un trabajo de recolección y análisis de datos que nos pemitió llegar a conclusiones sobre la hipótesis y a generalizaciones sobre los datos obtenidos, con referencia directa a la muestra estudiada.

La siguiente es una presentación sucinta de la investigación que realizamos.

\section{LA PRODUCCIÓN DEL LENGUAJE}

En términos generales, el proceso psicolingüístico de producción de enunciados en una lengua consiste en la aplicación de un conjunto de procesos y estrategias, unos y otras cognoscitivos, que convierten una intención comunicativa en una secuencia de sonidos o letras.

Teun van Dijk [1983:180] explica la diferencia entre procesos y estrategias de la siguiente forma:

Para entender adecuadamente la diferencia entre estrategias y reglas (procesos) puede observarse como ejemplo análogo el juego del ajedrez. En primer lugar existen reglas generales y fijas para jugar al ajedrez, reglas que en principio todo jugador debe observar si realmente quiere jugar. Pero además todo el sentido del juego consiste en que un jugador hace jaque mate al otro. Para conseguir este fin no sólo debe jugar "correctamente" el ajedrez sino también conoœr una serie de estrategias "óptimas" para hacelle jaque mate al otro.

Mientras que los procesos son generales y fijos, las estrategias varían constantemente, no sólo porque, a diferencia del ajedrez, los propósitos comunicativos son muchos, sino porque éstas dependen de una gran variedad de factores que fluctúan a menudo antes y durante toda interacción comunicativa.

\footnotetext{
${ }^{1}$ Decidimos responder la pregunta desde esta perspectiva, aunque hubiéramos podido escoger también el punto de vista de la recepción del lenguaje. La decisión fue un tanto arbitraria, por cuanto sólo tuvimos en cuenta nuestro interés personal por el tema de la producción del lenguaje.
} 
Los mecanismos cognoscitivos que operan durante la producción de enunciados, son ${ }^{2}$

1. La cons trucción de un propósito de comunicación a partir de las motivaciones y deseos del hablante.

2. La construcción del mensaje que se desea transmitir, a partir del conocimiento que el hablante tiene del mundo, real o posible.

3. La construcción de un significado literal o intención lingüística para expresar total o parcialmente el mensaje ya elaborado.

4. La planeación de una estructura que le dé forma lingüística al significado creado, a partir del conocimiento que el hablante posee de las reglas gramaticales de la lengua que va a utilizar.

\section{La ejecución motriz que convierte lo planeado en una secuencia sonora o} escrita.

El proceso global está presentado aquí como una secuencia de pasos. En realidad, existe evidencia de que cada proceso depende de los otros de manera interactiva ${ }^{3}$. Además, la secuencia de operaciones mecánicas está controlada en forma estratégica. Básicamente, existen dos tipos de estrategias que ejercen su influencia sobre la producción de enunciados:

1. Estrategia de comunicación. El objetivo de todo hablante es lograr su propósito comunicativo de la manera mas eficaz posible. Por tanto, todo el proceso, desde la construcción del mensaje, hasta la ejecución del enunciado, debe conducir a este objetivo. Para lograrlo, el hablante tiene en cuenta un conjunto de informaciones contextuales y selecciona el mensaje, el significado yla forma gramatical más apta para lograr la eficacia de su expresión.

2. Estrategia de corrección. El objetivo del hablante es lograr su propósito comunicativo en la forma más correcta posible. En consecuencia, durante las operaciones de selección de elementos en los distintos niveles del proceso, entra a funcionar una estrategia que evalúa los elementos evocados para deteminar si cl resultado es el más apropiado para su propósito comunicativo dentro de la situación en la que se encuentra.

${ }^{2}$ De las tres grandes áreas de investigación psicolingüística, a saber: la adquisición, la comprensión y la producción del lenguaje, esta última es la que se ha estudiado con menor exhaustividad. Sin embargo, existen ya algunas investigaciones sobre el tema. Autores como Garret, Fromkin, Dell, Kefler y Van Dijk, entre otros, han aportado elementos valiosos ara la construcción de una teoría psicolingii ística de la producción lingüística.

${ }^{3}$ Casi todos los modelos psicolingiiísticos de producción del lenguaje, propuestos hasta el momento, reproducen un proceso interactivo donde cada una de las fases afecta no solamente la que le sigue sino que puede influir sobre etapas anteriores. Ver Garnham [1985:215]. 
Cabe mencionar aquí una última diferencia entre procesos y estrategias de producción. Si bien los procesos son operaciones mentales automáticas, idénticas en todos los hablantes, de las estrategias se dice que pueden manipularse conscientemente por parte del hablante. Por tanto, cuando se afirma que una persona habla "mejor" que otra, nomalmente se concluye que la una maneja las estrategias psicolingüisticas mejor que la otra.

\section{HIPÓTESIS}

Ante la pregunta: ¿ qué entidad mental comporta aquello que en términos lingüís ticos y sociolingiístico llamamos la noma?, la consulta bibliográfica sobre el proceso de producción del lenguaje nos dio los medios para responder. Postulamos entonces, como hipótesis:

La norma se manifiesta psicológicamente en la aplicación, durante la producción del lenguaje, del proceso estratégico de evaluación del enunciado planeado o ejecutado.

Es durante la aplicación de la estrategia de corrección que el hablante maneja su conocimiento de las nomas aplicables al entomo comunicativo en el que se encuentra, para decidir si lo planteado o lo ejecutado es apropiado o no, y por ende, si el propósito comunicativo se logró en forma óptima o no.

Un fenómeno análogo se trabaja dentro de la teoría de adquisición de una segunda lengua. Allí, existe la noción del monitor, que es una especie de filtro por el que pasan todos los enunciados planteados por el aprendiz para que éste decida si lo que va a decir corresponde a las reglas de estructura y de uso de la lengua que está aprendiendo ${ }^{4}$.

Para poder validar empíricamente la hipótes is, era necesario encontrar la forma de "observar", al menos indirectamente, el funcionamiento interno de la estrategia de evaluación durante la producción de enunciados particulares. Tal problema se resolvió remitiéndonos a la posibilidad que tenemos los hablantes de auto corregimos cada vez que detectamos un "enror" en lo que decimos. Michael Stubbs [1987: 99] explica el hecho así:

... en el curso de la conversación natural se producen y corrigen errores. La posibilidad de corregirlos prueba la existencia de una norma. [...] Estos autores [...] han encontrado una preferencia general por la auto corrección; es decir, si los hablantes cometen algún tipo de error al hablar lo más probable es que se autccorrijan tanto si se dan cuenta de ello, como si se lo indica otro hablante.

Esta capacidad de autocorrección, reflejo directo de la existencia de nomas, nos permitió, por una parte, contrastar nues tra hipótes is con la realidad, y por otra,

\footnotetext{
${ }^{4}$ En su libro Understanding Second Language Acquisition

Rod Ellis explica ampliamente la noción del monitor para el proœso de aprendizaje de una segunda lengua.
} 
llegar a algunas generalizaciones sobre los tipos de noma que operan en el habla de un deteminado grupo de hablantes.

\section{MÉTODO}

A. Sujetos. - Trabajamos con el comportamiento lingüístico de una población constituida por hablantes nativos del es pañol. De dicha población, escogimos una muestra formada por estudiantes del Instituto Pedagógico Nacional, de Santafé de Bogotá, quienes estaban en los grados décimo y once, y cuyas edades oscilaban entre los 15 y 17 años. Seleccionamos estudiantes con miras a que las conclusiones a las que llegáramos sirvieran de base para consideraciones posteriores sobre la ens eñanza del español en el contexto escolar.

B. Procedimiento. - La recolección de datos se hizo mediante el procedimiento de observación no participante; los enunciados objeto de análisis se registraron mediante grabaciones magnetofónicas sin que se entrara en interacción comunicativa con los sujetos de la muestra.

Se llevaron a cabo 12 sesiones de grabación en el transcurso de clases regulares del colegio. Aunque las grabaciones también registraban el habla de los profesores, sus intervenciones no se tuvieron en cuenta para el análisis. Con posterioridad, tales grabaciones se transcribieron ortográficamente . sobre estas transcripciones se realizó el análisis.

C. Escenario. - Los datos que analizamos y clasificamos como material para la contratación de la hipótesis, fueron recolectados en el siguiente contexto o situación comunicativa:

\section{1.- Participantes.}

a. Hablantes. - Estudiantes de los grados décimo y once del Instituto Pedagógico Nacional.

b. Oyentes. - El profesor de la materia y los compañeros de clase.

\section{2. - Situación.}

a. Medio físico. - Un aula de clase con sus características típicas.

b. Período de tiempo. - Cada sesión de grabación duró aproximadamente 70 a 90 minutos, incluyendo las intervenciones del profesor y los períodos de silencio; así que, en realidad, el material analizado corresponde a cerca del 50 $\%$ del tiem po de grabación.

c. Patrones de comportamiento. - Se grabaron períodos de clase. Los sujetos producían enunciados como respuestas, comentarios o preguntas relativas al tema de la clase. En varias sesiones, se realizaron intervenciones 
expositivas por parte de los sujetos, sobre temas asignados previamente por el profesor.

3. Temas. - Las clases grabadas correspondieron a las asignaturas de Democracia (discurso de posesión del Presidente Gaviria, la Constitución de 1886 y la convocatoria a la Asamblea Nacional Constituyente) y Literatura (el nacimiento de la novela latinoamericana y el análisis de Don Juan Tenorio, de Zorrilla y La Colmena, de Camilo José Cela). Se grabó también una discusión sobre la experiencia de los alumnos en la presentación de los exámenes del ICFES.

4. Tipos de enunciados. - La situación y la misma naturaleza de los temas deteminaron que la mayoría de los enunciados analizados fuera de tipo declarativo: afirmaciones, argumentaciones, reiteraciones, etc. Tales declaraciones se producían como respuesta a preguntas o comentarios del profesor o como parte de una exposición preparada con anterioridad por el estudiante.

D. Variables.

1. Variable dependiente. - Según la hipótesis, la variable dependiente para nuestro análisis es el funcionamiento del proceso estratégico de evaluación del enunciado planeado o ejecutado.

Hemos dicho que este proceso opera siempre que se produce un enunciado; para el análisis que realizamos, esta variable sólo se observará en el momento en que ocurra una autocorrección en el habla del sujeto.

Definimos autocorrección de la siguiente forma:

Un enunciado presenta una autocorrección si dentro de él se observa que un elemento de contenido ocurre más de una vez, cambiando en su repetición uno o algunos de los siguientes aspectos: a) un elemento léxico, b) un elemento gramatical, c) su pronunciación, o d) la expresión completa.

2. Variable independiente. - De acuerdo con la hipótesis, la variable independiente corresponde a la acción de una de las normas, que hacen parte de la competencia comunicativa del hablante.

En términos de nuestro análisis, la variable independiente corresponde a la detección, durante el proceso de producción de unidades discursivas, de una violación de alguna de las nomas que están operando en el momento. Para el caso específico que analizamos, predecimos que tales normas son de tres tipos:

a. La noma lingüística: el proceso de evaluación trabajará sobre reglas gramaticales del sistema, que en este caso es el español. 
b. La noma social: para los sujetos de la investigación y para la situación concreta en que se obtuvieron los datos, el proceso de evaluación trabajará con el conjunto de normas que caracterizan el español estándar, de tipo académico, apropiado para los contenidos que se estaban trabajando en clase.

e. La noma socio-pragmática: en este caso, el proceso de evaluación trabajará con un conjunto de parámetros que $\mathrm{cl}$ individuo ha creado y que le permiten seleccionar, a su juicio, la forma más eficaz y apropiada para lograr su propósito comunicativo.

\section{ANÁLISIS}

A. Procedimiento. - El procedimiento de análisis comenzó con una revisión de las transcripciones ortográficas a fin de ubicar en ellas aquellos enunciados que contuvieran autocorrecciones. Una vez hecho ésto, elaboramos aparte una lista de los mismos. Posteriormente realizamos el análisis de los enunciados autocorregidos, categorizando cada uno según sistemas de clasificación previamente determinados. Luego, se hizo un sencillo estudio estadístico para ilustrar porcentajes de ocurrencia, y mostrar algunas correlaciones posibles. Por último, llegamos a conclusiones respecto de nuestra hipótesis y de otros aspectos relevantes para nuestra investigación.

B. Datos. - Identificamos un total de 302 enunciados autocorregidos. Probablemente algunas auto-correcciones no se hayan registrado como tales, ya sea porque existió duda para su identificación o porque la grabación no permitió comprender claramente lo que se decía.

Elaboramos tres sistemas de clasificación de los datos y analizamos cada una de las autocorrecctones detectadas desde estos tres puntos de vista a saber:

1. La descripción del fenómeno a partir del proceso de producción de enunciados. Encontramos que la evaluación produce su resultado concreto, una autocorrección, en dos momentos de la producción lingüística: antes o después de la etapa de ejecución del enunciado. Así, esta primera clasificación divide las autocorrecciones en dos grupos:

a. Aquellas que se producen cuando la estrategia de corrección detecta una violación de la noma antes de que se produzca la secuencia sonora. El hablante comienza a decir algo, se interrumpe y reinicia su intervención autocorrigiéndose. Un ejemplo de este tipo de autocorrección se observa en el enunciado

Por el, por la, por el surgimiento de la burguesía.

Notamos, por el sucesivo cambio de artículo, que el hablante duda en la selección léxica específica y se corrige antes de pronunciar la palabra que tiene en mente. En este caso específico, podría especularse que el hablante había planeado decir "la burguesía"; sin embargo, nunca se podrá saber exactamente qué palabra había seleccionado. 
b. Aquellas que se producen cuando la estrategia de corrección detecta una violación de la noma después de que se pronuncia la secuencia sonora. El hablante comienza a decir algo, terminama de decirlo y luego se autocorrige. Tenemos entonces enunciados como:

Sería una... desarrollo muy propio, ... muy autóctono de la novela donde observamos claramente qué elemento fue considerado "anormal" por el hablante.

Es importante mencionar aquí que las otras dos escalas de clasificación sólo se trabajaron para este segundo tipo de autocorrecciones. No fue posible analizar las primeras más profundamente, debido al riesgo de caer en especulaciones.

2. La descripción del fenómeno según el elemento sobre el cual operó el proceso de evaluación. En este caso, tenemos cuatro tipos de autocorrecciones:

a. Aquellas donde el elemento que se corrige es una expresión léxica: el reem plazo de una palabra por otra. Por ejemplo, "apoderarse" por "manejar" en:

Pues quería apoderarse eh. .. quería manejar esa situación.

b. Aquellas donde lo que se corrige es un elemento gramatical: como el cambio en la conjugación verbal que indica un cambio en la ubicación temporo-aspectual del evento al que se hace referencia, en:

En cada país se regresaban ... se regresaron a las costumbres..

c. Aquellas donde lo que se corrige es la pronunciación de lo planeado, cuando la articulación inicial se considera defectuosa. Por ejemplo:

Es un análisis his tórico de la novela late... latinoamericana.

d. Aquellas donde el elemento corregido es una frase o una oración completa: a este tipo de autocorrecciones corresponden los cambios de estructura gramatical (orden o función gramatical de los elementos). Como el paso de una oración impersonal a una con sujeto en primera persona:

Bueno, en lo que se ha podido ver ... pues en lo que he podido ver...

Incluimos también en esta categoría los cambios del contenido literal (aunque no intencional) de lo dicho; como, por ejemplo,

Para que deje su lucha violenta y sea ya una lucha... o sea..., que peleen por sus ideas pero que peleen de una forma pacífica.

3. La descripción del fenómeno según los tipos de noma que, de acuerdo a nuestra predicción, operan en el proceso de producción de enunciados. Este 
último análisis nos remite directamente a la hipótesis formulada; es aquí donde deteminamos más claramente su validez.

Encontramos que es posible ubicar cada enunciado autocorregido en alguna de las siguientes categorías:

a. Autocorrecciones producidas por acción de la noma lingüística: a esta categoría corresponden tanto los casos en los que se detecta una agramaticalidad, como los casos en los que se descubre que la forma seleccionada no transmite correctamente la intención lingüís tica cons truida. Por ejemplo:

Contra el lavado de dólar ... de dólares, la.. el tráfico de armas.

Doña Inés la presiona para pedir ... para eh! Brígida presiona a Doña Inés ...

b. Autocorrecciones producidas por acción de la noma social: tenemos aquí los casos en los que se detecta una ejecución inapropiada para la situación comunicativa. Por ejemplo:

Entonces.., que se querían venir hasta Bogotá para agarrar el poder, tomarlo ¿no?

El cambio léxico de "agarrar" a "tomar" se produce por la connotación poco formal del uso de la palabra "agarrar" en un salón de clase.

En el siguiente ejem plo observamos cómo dentro dc la noma social se incluyen expresiones léxicas impuestas por el texto al que se está haciendo referencia, en este caso, el Don Juan Tenorio, de Zorrilla:

Qué vio, que vio algo extraño en el jardín... en... el solar.

c. Autocorrecciones producidas por acción de la norma psico-pragmática: son aquellas en las que se detecta algo "anomal" en lo producido, a pesar de que el enunciado sea gramaticalmente correcto y apropiado al contexto com unicativo. Se producen entonces, cambios en el orden de los elementos que forman el enunciado, o cambios léxicos para clarificar la información, o variaciones morfológicas que cambian la modalidad del enunciado. Ejemplos de este tipo de autocorrección son:

Tú estabas hablando, tú estabas diciendo de...

Yo pensé que había ...... pudo haber tomado...

Entonces le dice ... él les empieza a decir que eso es una trampa.

C. Análisis estadístico. - Mediante diagramas se muestran las frecuencias de ocurrencia de las categorías identificadas en cada clasificación y la correlación entre las clasificaciones 2 y 3 . 


\section{Clasificación No. 1}

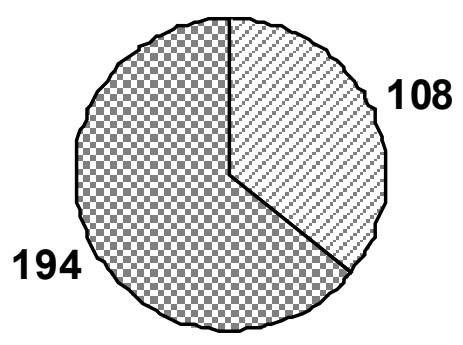

$\square$ ANTES 108

$37,5 \%$

口 DESPUES 194

$64,3 \%$

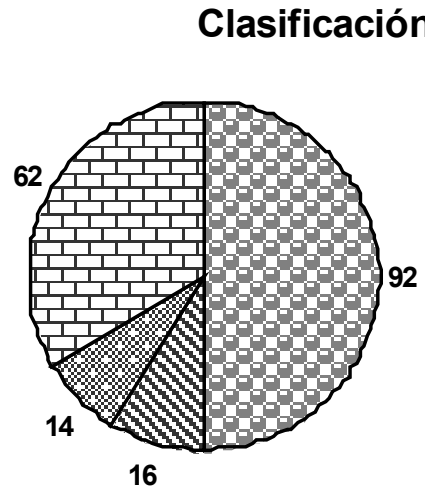

\begin{tabular}{|l|}
\hline 口LEXICO 92 \\
$47,4 \%$ \\
У MORFOL 26 \\
$13,4 \%$ \\
OFONETICO 14 \\
$7,2 \%$ \\
INTACT 62 \\
$32 \%$
\end{tabular}

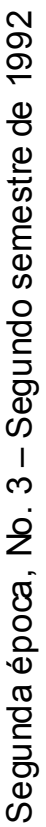

Clasificación No. 3

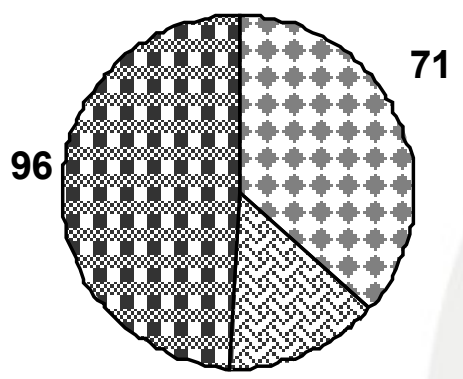

ㄴ LINGUIS 71
$36,5 \%$
O SOCIAL 37
$13,9 \%$
PSICO-PR 96
$49,6 \%$

27 


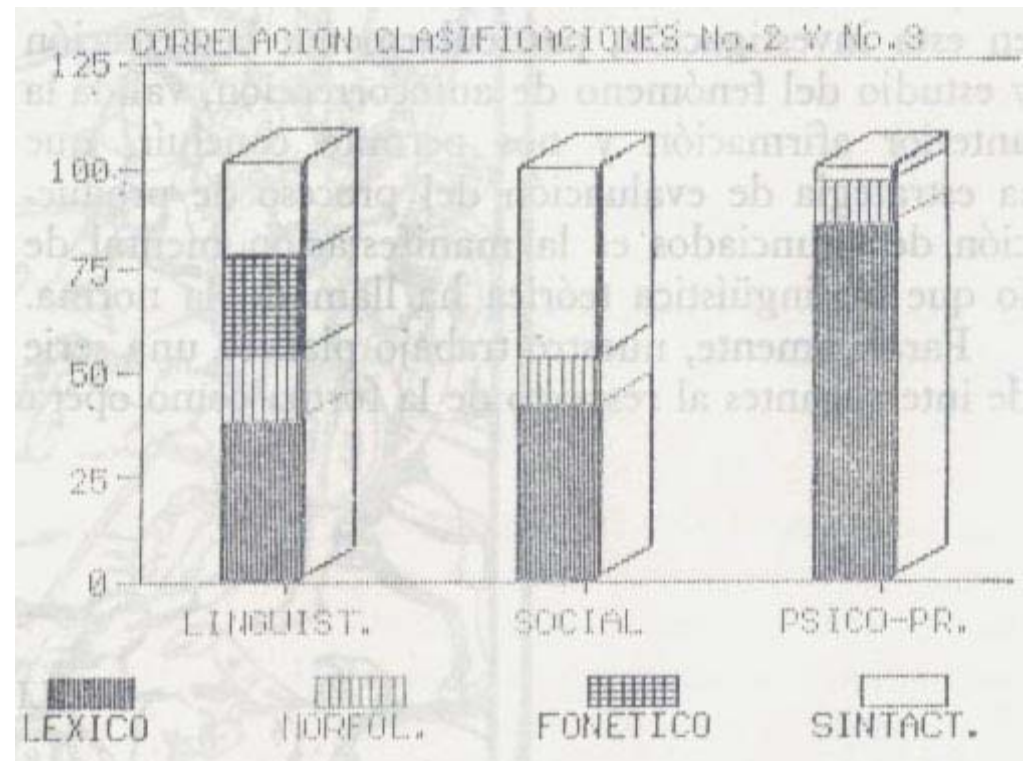

Estos cuadros nos permiten constatar lo siguiente:

Los cambios léxicos fueron, indudablemente, los mas frecuentes. Tal parece que existe mayor presión nomativa frente al tipo de vocabulario que el individuo usa, que frente al tipo de estructura sintáctica o morfológica que se escoja.

El tipo de noma que opero con mas frecuencia fue la psicopragmática. Esto nos remite a la distinción, explicada con anterioridad, entre mecanismos y estrategias de producción. Nos aventuramos a afimar que el grupo de individuos cuya habla analizamos, está aun en el proceso de aprendizaje de las estrategias comunicativas y su expresión no logra todavía altos niveles de efectividad. Existe, entonces un mayor grado de inseguridad en lo que se refiere al manejo de tales estrategias y uno menor para la puesta en práctica de las reglas (id sistema, ya que éstas operan de forma automática en los procesos mecánicos de producción de enunciados, y han sido aprendidas desde la niñez.

Observamos, sin embargo, una ocurrencia relativamente alta de autocorrecciones generadas por la noma lingüística. Se podría inferir que el hablante es inseguro en el manejo del código que está utilizando; sin embargo, hay que considerar que dentro de esta categoría están incluídas las autocorrecciones por la detección de contenidos erróneos y que sólo parte de ellas tienen lugar cuando ocurre un error gramatical. Por tanto, entran aquí en funcionamiento las variables de memoria, marco de conocimiento sobre el tema de la interacción comunicativa, etc. Un análisis posterior tendría que establecer la distinción entre estos tipos de auto-correcciones, puesto que es improbable que el hablante presente algún grado de inseguridad frente al conocimiento de las reglas gramaticales de su lengua materna. 


\section{CONCLUSIONES}

Una de las motivaciones que nos llevaron a iniciar la investigación que acabamos de describir, fue encontrar un punto convergente entre lo que la lingüística general, por un lado, y la psicolingüística, por el otro, han planteado sobre la producción del lenguaje.

Concentramos nuestras inquietudes en el aspecto del proceso sobre el cual ambas disciplinas han desarrollado aproximaciones teóricas: la evaluación del comportamiento lingüístico. El concepto de norma dentro de la lingüística general y la socio-lingüística y el proceso estratégico de supenisión del proceso de producción de enunciados, dentro de la psicolingüística, son nociones que se apoyan mutuamente a la hora de explicar la existencia de juicios por parte de los hablantes sobre la corrección o incorrección de un enunciado determinado.

El análisis del habla espontánea llevado a cabo en esta investigación, particularmente la detección y estudio del fenómeno de autocorrección, valida la anterior afimación y nos permite concluir, que la estrategia de evaluación del proceso de producción de enunciados es la manifestación mental de lo que la lingüís tica teórica ha llamado la noma.

Paralelamente, nuestro trabajo plantea una serie de interrogantes al respecto de la forma como opera la competencia comunicativa del hablante en el momento de la producción lingüística. Nos preguntamos, por ejemplo, si los diferentes tipos de noma -lingüística, social y psico-pragmática- ejercen su influencia sobre el proceso, con el mismo grado de intensidad o si existen niveles de influencia según la situación de comunicación o el tipo de acto de habla, o el estado psicológico del hablante. Asimismo, no está claro todavía qué factores generan la aparición de autocorrecciones en el habla de un individuo, puesto que és tas no se producen siempre que se comete un error.

Por último, a propósito de la utilidad que tenga un conocimiento profundo del proceso pslcolingüís tico de producción del lenguaje, para mejorar la enseñanza de la lengua materna, este trabajo abre caminos hacia investigaciones que aporten nuevos enfoques pedagógicos en este as pecto. Por ejemplo, un docente que sepa de la existencia del proceso estratégico de evaluación de enunciados y de su funcionamiento, puede echar mano del que ocurre en la mente de sus alumnos para crear en ellos la conciencia de reglas inmanentes en la lengua y su uso. Esto facilitaría en gran medida el desarrollo de las habilidades comunicativas de los estudiantes. Habría entonces que inves tigar cómo se desarrollaría tal enfoque. 


\section{BIBLIOGRAFÍA}

ARBOLEDA, RUBÉN, Las Normas Lingüísticas, en Revista Colombiana de Lingüística, núm. 6, Bogotá, junio 1986.

COSERIU, EUGENIO, Teoría del Lenguaje y Lingüística General, Madrid, Gredos, 1982.

DITTMAR, NORBERT, A Critical Survey of Sociolinguistics, New York, St. Martin's Press. 1976.

EYSENCK, MICHAEL \& KEANE, MARK, Cognitive Psychology, London, Lawrence Erbaum Associates, 1990.

GARNHAM, ALAN, Psycholinguistics, London, Methuen. 1985.

KELLER, ERIC, Intoduction aux System es Psycholinguistiques, Quebec, Gaetan Mourin Editeur, 1985.

STUBBS, MICHAEL, Análisis del Discurso, Análisis Socio-lingüístico del Lenguaje Natural, Madrid, Alianza Editorial, 1987.

TOBON DE CASTRO, LUCÍA, La ciencia Lingüística, una doble expectativa para el docente de lenguas, en Folios, Segunda época, num. 1, Bogotá, segundo semestre, 1990.

VAN DIJK, TEUN \& KINTSCH, WALTER, Strategis of Discourse Comprehension, New York, Academic Press, 1983. 